\title{
Medial Pterygoid Myositis Complicated by Numbness of the Tongue and Nonodontogenic Toothache: A Case Report
}

\author{
Takashi Uchida1, Takashi Iida' ${ }^{1}$, Osamu Komiyama ${ }^{1}$, Kayo Kuyama ${ }^{2}$ \\ ${ }^{1}$ Orofacial and Head Pain Clinic, Nihon University Hospital at Matsudo, Chiba, Japan \\ ${ }^{2}$ Department of Pathology, Nihon University School of Dentistry at Matsudo, Chiba, Japan \\ Email: uchida.takashi@nihon-u.ac.jp
}

How to cite this paper: Uchida, T., Iida, T., Komiyama, O. and Kuyama, K. (2020) Medial Pterygoid Myositis Complicated by Numbness of the Tongue and Nonodontogenic Toothache: A Case Report. Open Journal of Stomatology, 10, 241-249. https://doi.org/10.4236/ojst.2020.109023

\section{Received: July 31, 2020}

Accepted: September 11, 2020

Published: September 14, 2020

Copyright $\odot 2020$ by author(s) and Scientific Research Publishing Inc. This work is licensed under the Creative Commons Attribution International License (CC BY 4.0).

http://creativecommons.org/licenses/by/4.0/

\begin{abstract}
A 34-year-old woman visited our clinic with complaints of trismus, numbness in the left half of the tongue, and pain in tooth 34 . At the patient's initial visit to our clinic, the maximum assisted jaw opening movement was $20 \mathrm{~mm}$, pointing to severe trismus. The patient complained of spontaneous pain in tooth 34 but did not evince percussion pain. Her pain remained unchanged even under local anesthesia. Radiography showed no pathological findings. A tenderness test of masticatory muscles failed to induce pain. The presence of severe trismus argued against temporomandibular disorders, resulting in suspicion of other inflammatory disease. In view of numbness of the tongue, other diseases such as inflammation or neoplastic disease in the head and neck region were considered. The patient was referred to the departments of neurosurgery and otolaryngology for examination. The results of MRI and CT diagnosis led to the identification of acute myositis of the left medial pterygoid muscle requiring the prescription of nonsteroidal anti-inflammatory drugs, and relaxation of mandibular muscles. With time, maximum unassisted jaw opening improved and pain in tooth 34 ceased, but tongue numbness persisted. The condition was attributed to compression of lingual nerve (LN) and mandibular nerve (MN) resulting from medial pterygoid muscle inflammation.
\end{abstract}

\section{Keywords}

Medial Pterygoid Muscle, Mandibular Nerve, Lingual Nerve, Referred Pain

\section{Introduction}

Toothache, the most common type of orofacial pain [1], is often diagnosed as 
odontogenic. However, toothache also manifests as nonodontogenic pain without odontogenic origin [2]. Among cases of nonodontogenic toothache, neuropathic pain in the orofacial region is often induced by direct compression of the trigeminal nerve and can cause heterotopic pain [3]. Three kinds of heterotopic pain are described: central pain, projected pain and referred pain [2]. In cases of central pain, a lesion or event within the brain or brain stem may damage nerve tissue. As a consequence, pain may be experienced in the oral-facial region. When a nerve is irritated or triggered at some point of its anatomical trajectory, the pain sensation follows the course of nerve branch,which is called a projected pain. The third kind of referred pain is central sensitisation, which is considered a key factor in the genesis and continuation of chronic pain [4]. Heterotopic pain in the orofacial region is, in most cases, difficult for dentists to make an accurate diagnosis. Diagnosis is even more difficult in cases in which patients present with multiple symptoms.

In this paper, the authors report a case in which the patient's condition (i.e., trismus, toothache, and numbness of the tongue) was determined to be attributable to compression of the lingual nerve (LN) and mandibular nerve (MN) due to inflammation of the medial pterygoid muscle.

\section{Case Report}

A 34-year-old female visited our Orofacial and Head Pain Clinic with a chief complaint of spontaneous pain in the left lower back teeth. At one week before her initial visit to our hospital in year $\mathrm{X}$, she had experienced spontaneous pain in the region of the left mandibular molar teeth. While this was followed by trismus due to mandibular pain, the patient's toothache abated. At one day before the patient's initial visit in year X, pain in the left mandibular molar teeth recurred, along with numbness in the left half of the tongue. The patient visited our hospital in year X. Severe trismus was noted at the initial visit, as indicated by both the maximum unassisted and assisted jaw opening movements being 20 $\mathrm{mm}$. Tenderness testing identified tenderness of the bilateral masseter muscle, left temporalis, and left lateral pterygoid muscle. A fixed prosthodontic restoration was present in the region of the right mandibular molar teeth. Although the patient complained of spontaneous pain in tooth 34, no pathological findings were macroscopically present, nor was percussion reaction present (Figure 1).

Radiography showed no pathological findings (Figure 2, Figure 3). Additionally, although the patient complained of numbness in the left half of the tongue, neither macroscopically pathological findings nor palpation-induced pain was present. Based on the patient's symptoms and laboratory findings, we considered a diagnosis of dysesthesia.

Tendency toward depression was weak based on the result of patient health questionnaire (PHQ)-9; anxiety tendency was absent on the General Anxiety Disorder (GAD)-7 scale; and the PHQ-15 result indicated moderate tendency toward somatization. These findings suggested a low likelihood of psychosocial pain. 


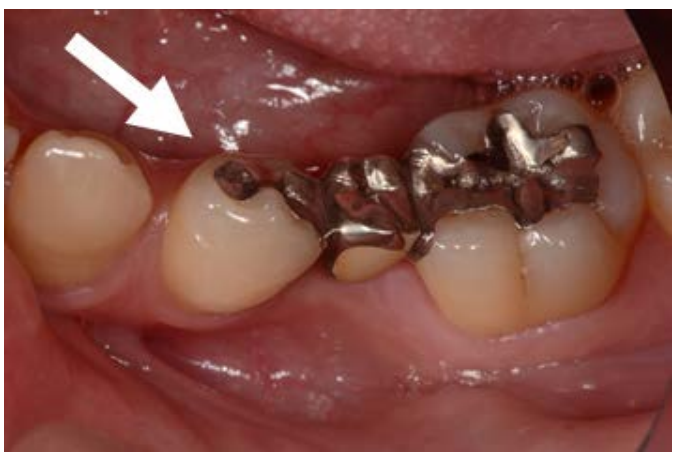

Figure 1. Intraoral photograph: no macroscopically pathological findings observed in tooth 34 , adjoining teeth, and periodontal tissue.

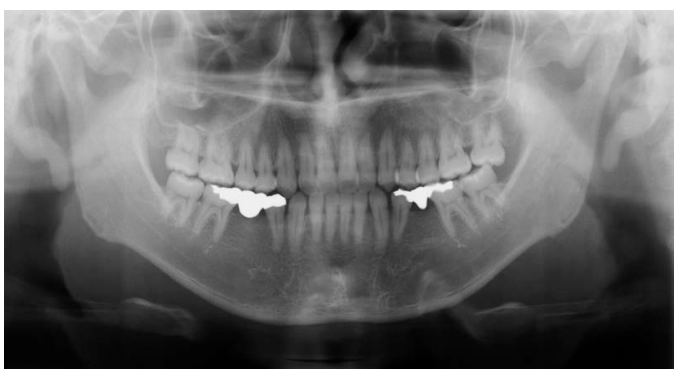

Figure 2. Panoramic radiograph: adjoining teeth, opposing teeth, and surrounding tissues, including tooth 34 , showed no pathological findings observed by radiography.

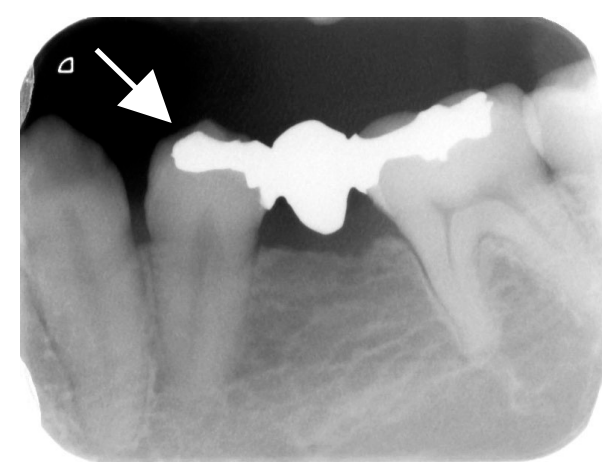

Figure 3. Oral radiograph: tooth 34 , adjoining teeth, and periodontal tissue showed no pathological findings observed by radiography.

A tenderness test of the left masseter muscle did not induce pain in tooth 34 . Despite anesthesia of the dental root membrane of tooth 34 , trismus and toothache remained unchanged.

The presence of severe trismus raised the suspicion of inflammatory disease other than temporomandibular disorders (TMD). Other diseases, such as inflammation and neoplastic disease in the head and neck region, were also considered in the presence of numbness of the tongue. We asked the department of neurosurgery to perform an examination 4 days after the patient's initial visit in 
year X. At the department of neurosurgery, the patient underwent blood testing and magnetic resonance imaging (MRI) examination. Blood testing revealed normal levels of C-reactive protein (CRP) and soluble interleukin (IL)-2 receptor. T2-weighted fat saturated STIR MRI revealed a non-uniform signal anomaly region of moderate to high signal intensity in the medial pterygoid muscle of the left masticator space (Figure 4).

While the anomaly region was adjacent to the cortical bone of the left mandibular branch, no abnormal signals in the bone marrow were found. These findings suggested a high likelihood of inflammation of the medial pterygoid muscle. We then referred the patient to the department of otolaryngology for examination 11 days after the patient's initial visit in year X. The results revealed a red swelling in the left pharyngeal region. An interview of the patient at the time revealed that the toothache manifested after she ate hard food. Computed tomography (CT) performed to examine bone tissue revealed greater swelling of the medial pterygoid muscle on the left side than on the right side, without no abnormalities in the bone tissue (Figure 5).

The patient was diagnosed with acute myositis of the left medial pterygoid muscle due to trauma attributable to the mastication of hard food. In addition to the above examinations, the patient was instructed to relax the mandibular muscles. Antibiotic and nonsteroidal anti-inflammatory drugs were prescribed. A second blood testing performed 11 days after the patient's initial visit in year $\mathrm{X}$ revealed normal values of CRP, soluble IL-2 receptor, and SCCM. At a follow-up visit 40 days after the initial visit in year $\mathrm{X}$, the maximum unassisted jaw opening movement was $58 \mathrm{~mm}$. Additionally, we found no signs of inflammation in the pharyngeal region. The numbness of the tongue improved, although failing to resolve completely. The pain in tooth 34 had disappeared. Therefore, treatment was tentatively completed and the patient was instructed to call back in the event of recurring symptoms in the follow-up period.

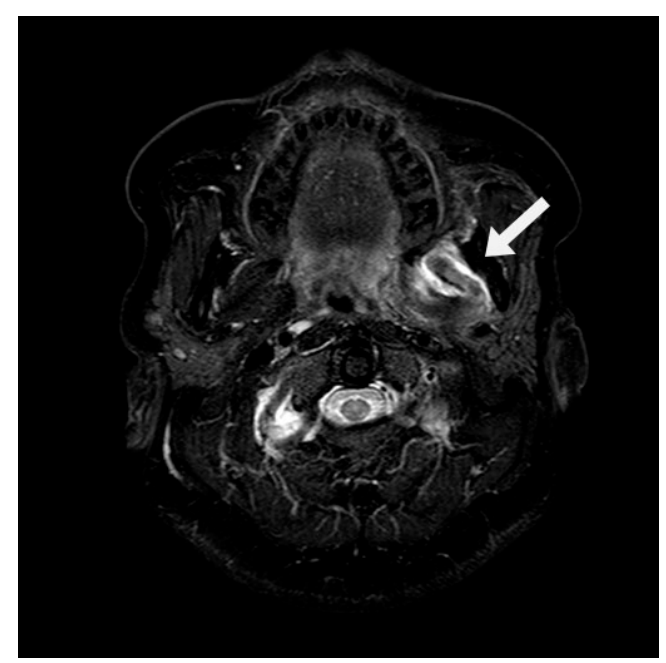

Figure 4. T2-weighted fat saturated STIR MRI (axial section). A non-uniform signal anomaly region of moderate to high signal intensity is observed in the medial pterygoid muscle of the left masticator space. 


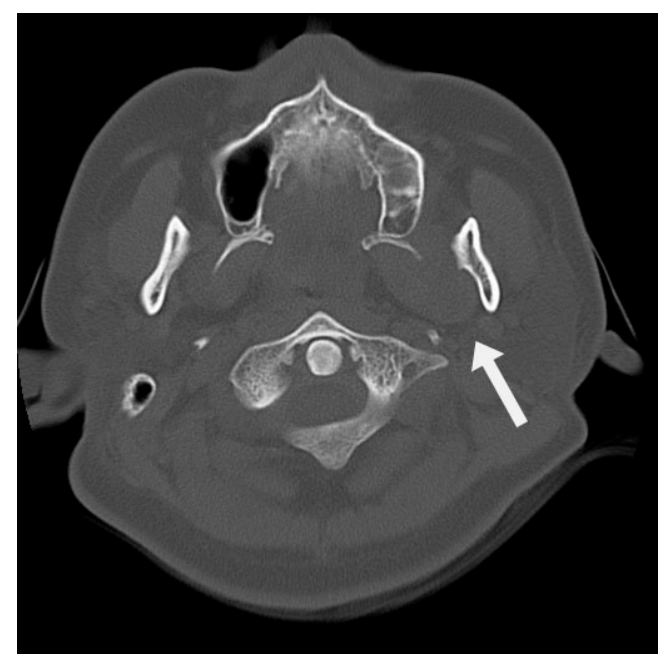

Figure 5. Computed tomography image (axial section). More pronounced swelling is found in the left than in the right medial pterygoid muscle, but no abnormal findings are observed in the bone tissue.

\section{Discussion}

The patient's chief complaint was pain in tooth 34 . Neither intraoral examination nor radiography revealed pathological findings for tooth 34 . Local anesthesia of the dental root membrane for tooth 34 had no effect on the toothache. Based on these findings, the pain in tooth 34 was diagnosed as nonodontogenic toothache without odontogenic origin. Myofascial pain referred to tooth (MPRT), the most common nonodontogenic toothache, is often noted in the masseter muscle as the causative muscle of MPRT [5]. Toothache manifesting as referred pain is observed in both the maxillary and mandibular teeth [6]. In the case of our patient, since tenderness was noted in the bilateral masseter muscle, MPRT originating in the left masseter muscle was suspected. However, continuing pain in tooth 34 observed in a tenderness test refuted the possibility of MPRT. Referred pain due to muscular disorders in the medial pterygoid muscle reportedly occurs in the pharynx and the posterior part of the mandible, not in the teeth [6], although we could not confirm this because of the difficulty of performing an accurate tenderness test of the medial pterygoid muscle. Her severe trismus was not odontogenic but appeared attributable to inflammation or a tumor in the head and neck region. Treatment for trismus was therefore prioritized.

Fairly often, we encounter cases of trismus in our daily dental practice. Many cases are associated with TMD [7]. Since trismus is associated with various disorders, such as inflammation, tumors, and scar contracture, dentists often encounter cases in which accurate diagnosis is difficult. In the case of our patient in particular, since the maximum movements of both assisted and unassisted jaw opening were $20 \mathrm{~mm}$, some disease other than TMD appeared involved in the development of trismus. We performed an MRI examination, which revealed abnormalities in the left medial pterygoid muscle. 
MRI is useful for ascertaining abnormal findings suggestive of inflammatory diseases of inside tissue (e.g., the medial pterygoid muscle) [8]. Kamio et al. [7] used MRI to examine the head and neck region in patients with suspected TMD. They observed clinical findings and signs similar to TMD, such as pain and trismus, in 21 patients, more than half of whom presented with mandibular osteomyelitis. Since radiography is not helpful in diagnosing osteomyelitis in the early stages, MRI is reported to be useful. In the case of our patient, normal CRP levels argued against severe inflammatory disease. Therefore, MRI screenings for detailed examination were performed only in the department of neurosurgery. Cancer of the head and neck region infiltrates the masticatory muscles with development of the cancer, reducing the range of jaw opening movement. In patients with trismus associated with a tumor in the oral cavity or pharynx, it is difficult to determine using imaging tests whether the tumor directly infiltrates the masticatory muscles [9]. However, blood testing showed that the soluble IL-2 receptor level of our patient was normal. MRI examination is important in the diagnosis of neoplastic disease, like in cases of osteomyelitis, when presenting with symptoms similar to those of TMD [10]. For patients presenting with TMD symptoms, the possibility of inflammation and tumors should be considered [11]. However, in the case of our patient, the findings of MRI and blood testing indicated such neoplastic disease was unlikely. Trauma of the medial pterygoid muscle may cause myositis ossificans traumatica (MOT). The onset of MOT in the medial pterygoid muscle is said to be rare [12]. Previous studies report have suggested that the cause of the MOT was profound trauma [13] [14] [15] [16]. There are no descriptions of trauma caused solely by mastication of hard food. MOT is believed to occur in association with intramuscular bleeding after trauma [17], although the mechanism remains unknown. In the case of our patient, we observed no trauma indicative of intramuscular bleeding, nor did we suspect infection. In addition, acute changes associated with trismus due to trauma to the medial pterygoid muscle are reported to subside rapidly [8]. It is unlikely that our patient would progress to MOT in the future. If the trismus recurs, a re-examination that accounts for the possibility of MOT would be necessary.

Anatomically, the MN is likely to be compressed by other tissue [18]. When the $\mathrm{LN}$, a main branch of the middle ear nerve, is compressed, numbness of the tongue, hypogeusia, tongue paralysis, dysgeusia of the anterior two thirds of the tongue, paralysis of the lingual gingiva, pain, and dysphonia may occur [19]. Our patient complained of numbness in the left half of the tongue. The LN generally runs on the outer surface of the medial pterygoid muscle. However, it can also penetrate the medial pterygoid muscle [20], which may lead to neuralgia of unknown cause [21]. In the case of our patient, since we did not suspect neoplastic disease, numbness of the tongue may have developed via the following mechanism: acute inflammation in the medial pterygoid muscle due to trauma causes swelling, which compresses the LN running on the outer surface of the medial pterygoid muscle or penetrating the medial pterygoid muscle, resulting in numbness of the tongue. The medial pterygoid muscle is situated medial to 
the MN. Medial pterygoid muscle located medial side of mandibular nerve. The central side of the mandibular foramen of the mandibular nerve is located between the medial pterygoid muscle and the mandibular branch [22]. Thus, swelling of the medial pterygoid muscle may also have compressed the $\mathrm{MN}$, as with compression of the LN, between the medial pterygoid muscle and the mandibular branch, resulting in referred pain in the $\mathrm{MN}$ region and, finally, projected pain in tooth 34 [2].

\section{Conclusion}

Diagnoses for abnormalities involving the LN require attention to the compression of nerves and blood vessels in the region where the $\mathrm{LN}$ is distributed. Similarly, nonodontogenic toothache in the region involving the $\mathrm{MN}$ requires careful diagnosis. An MRI examination of our patient revealed that multiple abnormalities, including symptoms due to compression of the $\mathrm{LN}$ and the MN, as well as trismus were attributable to inflammatory swelling of the medial pterygoid muscle. Diagnosis of multiple abnormalities in the oral and facial regions is often difficult. We found MRI is effective in diagnosing head and neck region diseases and that active use of MRI can provide a more reliable diagnosis.

\section{Acknowledgements}

We wish to express our gratitude to Professor Yasuhide Makiyama and Associate Professor Hideo Niwa of the Department of Neurosurgery/Head and Neck Surgery, Nihon University School of Dentistry at Matsudo, for their cooperation and advice regarding treatment.

\section{Consent}

The patient has given her consent for this report to be published and it is in her chart at the Nihon University Hospital at Matsudo.

\section{Conflicts of Interest}

The authors declare no conflicts of interest regarding the publication of this paper.

\section{References}

[1] Okeson, J. (2014) Bell's Oral and Facial Pain. 7th Edition, Quintessence, Chicago.

[2] De Laat, A. (2020) Differential Diagnosis of Toothache to Prevent Erroneous and Unnecessary Dental Treatment. Journal of Oral Rehabilitation, 47, 775-781. https://doi.org/10.1111/joor.12946

[3] Noma, N., Hayashi, M., Kitahara, I., Young, A., Yamamoto, M., et al. (2017) Painful Trigeminal Neuropathy Attributed to a Space-Occupying Lesion Presenting as a Toothache: A Report of 4 Cases. Journal of Endodontics, 43, 1201-1206. https://doi.org/10.1016/j.joen.2017.02.002

[4] Sessle, B.J. (2000) Acute and Chronic Craniofacial Pain: Brainstem Mechanisms of Nociceptive Transmission and Neuroplasticity, and Their Clinical Correlates. Criti- 
cal Reviews in Oral Biology \& Medicine, 11, 57-91. https://doi.org/10.1177/10454411000110010401

[5] Wright E.F. (2000) Referred Craniofacial Pain Patterns in Patients with Temporomandibular Disorder. Journal of the American Dental Association, 131, 1307-1315.

[6] Simons, D.G. and Travell, J.G. (1983) Myofascial Pain and Dysfunction: The Trigger Point Manual. 1st Edition, Williams \& Wilkins, Baltimore.

[7] Kamio, T., Yakushiji, T., Takaki, T., Shibahara, T., Imoto, K., et al. (2019) Incidental findings during Head and Neck MRI Screening in 1717 Patients with Temporomandibular Disorders. Oral Radiology, 35, 135-142. https://doi.org/10.1007/s11282-018-0327-y

[8] Ciacchella, A.P. and Higgins, W.L. (1990) MR Imaging of Pterygoid Muscle Inflammation. Magnetic Resonance Imaging, 8, 665-667. https://doi.org/10.1016/0730-725X(90)90148-U

[9] Satheeshkumar, P.S., Mohan, M.P. and Jacob, J. (2014) Restricted Mouth Opening and Trismus in Oral Oncology. Oral Surgery, Oral Medicine, Oral Pathology, and Oral Radiology, 117, 709-715. https://doi.org/10.1016/j.0ooo.2014.02.031

[10] Alvarez-Arenal, A., Gonzalez-Gonzalez, I., Moradas Estrada, M., de Llanos-Lanchares, H. and Costilla-Garcia, S. (2016) Temporomandibular Disorder or Not? A Case Report. Cranio, 34, 264-269. https://doi.org/10.1179/2151090315Y.0000000018

[11] Heo, M.S., An, B.M., Lee, S.S. and Choi, S.C. (2003) Use of Advanced Imaging Modalities for the Differential Diagnosis of Pathoses Mimicking Temporomandibular Disorders. Oral Surgery, Oral Medicine, Oral Pathology, and Oral Radiology, 96, 630-638. https://doi.org/10.1016/S1079-2104(03)00373-1

[12] Thangavelu, A., Vaidhyanathan, A. and Narendar, R. (2011) Myositis Ossificans Traumatica of the Medial Pterygoid. International Journal of Oral and Maxillofacial Surgery, 40, 545-549. https://doi.org/10.1016/j.ijom.2010.10.024

[13] Hanisch, M., Hanisch, L., Fröhlich, L.F., Werkmeister, R. and Bohner, L. (2018) Myositis Ossificans Traumatica of the Masticatory Muscles: Etiology, Diagnosis and Treatment. Head \& Face Medicine, 14, Article No. 23. https://doi.org/10.1186/s13005-018-0180-6

[14] Boffano, P., Zavattero, E., Bosco, G. and Berrone, S. (2014) Myositis Ossificans of the Left Medial Pterygoid Muscle: Case Report and Review of the Literature of Myositis Ossificans of Masticatory Muscles. Craniomaxillofacial Trauma and Reconstruction, 7, 43-50. https://doi.org/10.1055/s-0033-1356760

[15] Reddy, S.P., Prakash, A.P., Keerthi, M. and Rao, B.J. (2014) Myositis Ossificans Traumatica of Temporalis and Medial Pterygoid Muscle. Journal of Oral and Maxillofacial Pathology, 18, 271-275. https://doi.org/10.4103/0973-029X.140781

[16] Choudhary, A.K., Sahoo, N.K. and Chattopadhyay, P.K. (2012) Myositis Ossificans Traumatica of the Medial Pterygoid Muscle: A Case Report. Journal of Oral and Maxillofacial Surgery, Medicine, and Pathology, 24, 241-244.

https://doi.org/10.1016/j.ajoms.2011.12.004

[17] Rattan, V., Rai, S. and Vaiphei, K. (2008) Use of Buccal Pad of Fat to Prevent Heterotopic Bone Formation after Excision of Myositis Ossificans of Medial Pterygoid Muscle. Journal of Oral and Maxillofacial Surgery, 66, 1518-1522. https://doi.org/10.1016/j.joms.2007.05.020

[18] Piagkou, M., Demesticha, T., Skandalakis, P. and Johnson, E.O. (2011) Functional Anatomy of the Mandibular Nerve: Consequences of Nerve Injury and Entrapment. Clinical Anatomy, 24, 143-150. https://doi.org/10.1002/ca.21089 
[19] Piagkou, M.N., Demesticha, T., Piagkos, G., Androutsos, G. and Skandalakis, P. (2011) Mandibular Nerve Entrapment in the Infratemporal Fossa. Surgical and Radiologic Anatomy, 33, 291-299. https://doi.org/10.1007/s00276-010-0706-5

[20] Sakamoto, Y. and Akita, K. (2004) Spatial Relationships Between Masticatory Muscles and Their Innervating Nerves in Man with Special Reference to the Medial Pterygoid Muscle and Its Accessory Muscle Bundle. Surgical and Radiologic Anatomy, 26, 122-127. https://doi.org/10.1007/s00276-003-0177-Z

[21] Shimokawa, T., Akita, K., Sato, T., Ru, F., Yi, SQ., et al. (2004) Penetration of Muscles by Branches of the Mandibular Nerve: A Possible Cause of Neuropathy. Clinical Anatomy, 17, 2-5. https://doi.org/10.1002/ca.10169

[22] Akita, K., Sakaguchi-Kuma, T., Fukino, K. and Ono, T. (2019) Masticatory Muscles and Branches of Mandibular Nerve: Positional Relationships between Various Muscle Bundles and Their Innervating Branches. The Anatomical Record (Hoboken), 302, 609-619. https://doi.org/10.1002/ar.23943 